\title{
Cross-national analysis about the difference of histopathological management in Tis and T1 colorectal cancer between Japan and Korea
}

\author{
Naohisa Yoshida ${ }^{1)}$, Yuji Naito ${ }^{1)}$, Yutaka Inada ${ }^{1)}$, Yoshito Itoh ${ }^{1)}$, Sang Pyo Lee ${ }^{2)}$, Jeong Hwan Kim²), In-Kyung Sung ${ }^{2}$, \\ Hyung Seok Park ${ }^{2}$, Hye Seung Han ${ }^{3)}$, Masayoshi Nakanishi ${ }^{4)}$, Mitsuo Kishimoto ${ }^{5}$ and Sun-Young Lee ${ }^{2)}$ \\ 1) Department of Molecular Gastroenterology and Hepatology, Kyoto Prefectural University of Medicine, Kyoto, Japan \\ 2) Department of Internal Medicine, Konkuk University School of Medicine, Seoul, Korea \\ 3) Department of Pathology, Konkuk University School of Medicine, Seoul, Korea \\ 4) Department of Surgery, Kyoto Prefectural University of Medicine, Kyoto, Japan \\ 5) Department of Surgical Pathology, Kyoto Prefectural University of Medicine, Kyoto, Japan
}

\begin{abstract}
:
Objectives: There are differences in each country with regards to histopathological managements of colorectal cancer (CRC), such as definition of Tis and lymphatic and venous invasion. In this study, we compared Tis and T1 CRC in Japan and Korea. Methods: We retrospectively compared various clinical characteristics of consecutive patients who had Tis and T1 CRCs and who were newly diagnosed between 2010 and 2014 at the Kyoto Prefectural University of Medicine (Japan) and the Konkuk University (Korea). Results: Three hundred and sixty-five cases of T1 cancer and 510 cases of Tis cancer from 726 Japanese and 149 Korean patients were included. The rate of Tis in Japan was higher than in Korea (59.8\% vs. 51.0\%, $P$ $=0.047$ ), according to the difference of definition of Tis. In the analyses of 365 T1 CRCs, median age was higher in Japan than Korea $(67.8 \pm 10.6$ vs. $62.2 \pm 10.1, P<0.001)$. Right-sided lesions were more frequent in Japan than they were in Korea (38.7\% vs. $22.2 \%, P<0.001)$. The rates of venous and lymphatic invasion were higher in Japan than they were in Korea (venous: $18.6 \%$ vs. $1.4 \%, P<0.001$, lymphatic: $25.3 \%$ vs. $13.7 \%, P=0.042$ ), according to the different methods of immunohistochemical examinations used (Japan: E-HE and D2-40, Korea: ERG). Conclusions: Our study of T1 CRC showed that there were differences between Japan and Korea in tumor location, elderly incidence, and histopathological lymphatic and venous invasion. Additionally, rates of Tis were different between the two countries. In this international study for CRC, it is considered that we have to pay attention regarding the difference of histopathological definition and method in each country.
\end{abstract}

Keywords:

T1 cancer, colorectal cancer, Korea, Japan

J Anus Rectum Colon 2019; 3(1): 18-26

\section{Introduction}

In East Asia, including in Japan and Korea, incidence of colorectal cancer (CRC) is rapidly increasing, and in 2012 Korea had the highest incidence rate of CRC in the world ${ }^{1,2)}$. In Japan, CRC in 2017 was the first leading cause of cancer incidence $(149,500$ out of 124 million people) and the second leading cause of death to cancer $(53,000 \text { people })^{3)}$. However, in Korea, CRC was the third leading cause of death due to cancer in 2014 (26,978 out of 51 million people), and thyroid cancer and gastric cancer were the first and the second ${ }^{4}$. Generally, CRC incidence increases with age, 
and population aging is proceeding rapidly in Japan and Korea. In 2015, more than 33 million Japanese people were more than 65 years old, indicating an increase in the incidence of CRC in elderly people (26.8\% of the total population) compared with $14.5 \%$ of the total population in $1995^{5)}$. On the other hand, in Korea, $13.1 \%$ of people were more than 65 years old in 2015, compared with $5.0 \%$ in $1995^{6}$. Therefore, an overall aging population could be one reason for both countries for the increased incidence of CRC. However, there are lots of differences about the factors related to CRC in each country. For example, there are differences in food preferences, culture, CRC screening method and response rate, endoscopic diagnosis, and histopathological evaluations. Over recent decades, Korean and Japanese food has changed from a traditional rice-based diet to a Westernized one. In Korea, people seem to prefer consuming meat more than Japan. According to the 2015 Korea National Health and Nutrition Examination Survey, the average Korean consumes approximately $109.4 \mathrm{~g}$ of red meat per day, which is nearly three times higher than rates seen 30 years $\mathrm{ago}^{7}$. On the other hand, a Japanese study showed that average red meat consumptions of Japanese people were 51.5$68.4 \mathrm{~g} /$ day for men and 19.8-28.1 g/day for women ${ }^{8}$. Red meat and processed meat were reported to be associated with an increased risk of $\mathrm{CRC}^{9}$. Additionally, method of CRC screening in both countries is done by fecal immunochemical test (FIT). However, the starting age for screening is older in Korea (at least 50 years old) compared with Japan (at least 40 years old). The response rate for FIT is higher in Korea (44.7\% in 2012) than it is in Japan (Male: $41.4 \%$, Female: $34.5 \%$ in 2013$)^{1,10)}$.

Improvements in endoscopic diagnosis, such as narrow band imaging (Olympus Co.), blue laser imaging (Fujifilm), and pit pattern observation enable us to diagnose CRC accurately ${ }^{11-13}$. Additionally, endoscopic resections such as endoscopic submucosal dissection and endoscopic mucosal resection for Tis and part of $\mathrm{T} 1$ cancer have also improved significantly such that we can now resect large tumors without surgical operation ${ }^{14,15}$. Because of these improvements, initial therapeutic methods for early colorectal cancer (CRC) have shifted to endoscopic resection, especially for the elderly, because endoscopic resection is less invasive than traditional surgical operation ${ }^{16}$. However, these endoscopic diagnoses and therapies vary in each country. Additionally, there are differences regarding histopathological managements of CRC, such as definition of Tis and lymphatic and venous invasion in each country. In order to decrease CRC deaths in the world, analysis of characteristic differences of CRC in more than two countries is necessary. However, limited cross-national studies about CRC have been performed. In this study, we compared Tis and T1 CRC characteristics in two countries, Korea and Japan, with a particular focus on histopathological management of T1 CRCs.

\section{Methods}

This study was a multicenter retrospective study that assessed 875 consecutive patients (726 Japanese cases and 149 Korean cases) with Tis and T1 cancer between January 2010 and December 2014 at the Kyoto Prefectural University of Medicine (Kyoto, Japan) and the Konkuk University (Seoul, Korea), which included Tis and T1 cancers, and who were newly diagnosed with adenocarcinoma. The inclusion criteria were tumors that were resected by endoscopic resection or surgical operation and were histopathologically diagnosed as Tis or T1 CRCs at each institution.

By reviewing medical records, we analyzed in both countries overall various clinical factors and outcomes of Tis and T1 CRCs, including age, rate of elderly people ( $\geq 65$ years old), rate of number of people $\geq 75$ years old, sex, tumor size, tumor location, rate of antithrombotic use, initial therapeutic method (surgery or endoscopy), histological type (cell type), invasion depth (Tis or T1), and complications. With respect to the difference between Tis and T1 CRCs, the various clinical characteristics in both countries were also analyzed in Tis and T1 CRCs, respectively. Regarding T1 cancer receiving endoscopic surgery, the rates of additional surgery were analyzed in both countries. The indications of the additional surgery were poor histology, lymphatic and venous invasion, deeper submucosal invasion, and grade $2 \geq$ budding, according to Japanese guidelines in both countries $^{14)}$. Additionally, detailed analysis of tumor location of T1 CRCs in the elderly and the non-elderly in the two countries were analyzed. Moreover, multivariate analysis was performed to analyze related factors of right-sided colon location compared to other locations in T1 CRCs.

Tumor location was classified using three segments, which included the right-sided colon (from the cecum to the transverse colon), the left-sided colon (from the descending colon to the sigmoid colon), and the rectum. Complications, which included postoperative hemorrhage, perforation, infection, anastomotic leak, pancreatic fistula, and pulmonary dysfunction, were examined both after endoscopic resection and after surgical operation. Perforation was detected using endoscopy or abdominal computed tomography. Postoperative hemorrhage was defined as the occurrence of hematochezia requiring endoscopic and radiologic treatment to stop bleeding.

Histopathological diagnoses were performed by two clinical pathologists (A.Y. and M.K.) in Japan and one pathologist (H.S.) in Korea. In Korea, these diagnoses were performed according to the World Health Organization classification, and in Japan it was performed according to the Japanese Classification of Colorectal Carcinoma proposed by the Japanese Society for Cancer of the Colon and Rectum ${ }^{17,18}$. All tumors were classified as Tis or T1 CRCs. The definition for Tis was severe cytologic dysplasia, structural atypia, 

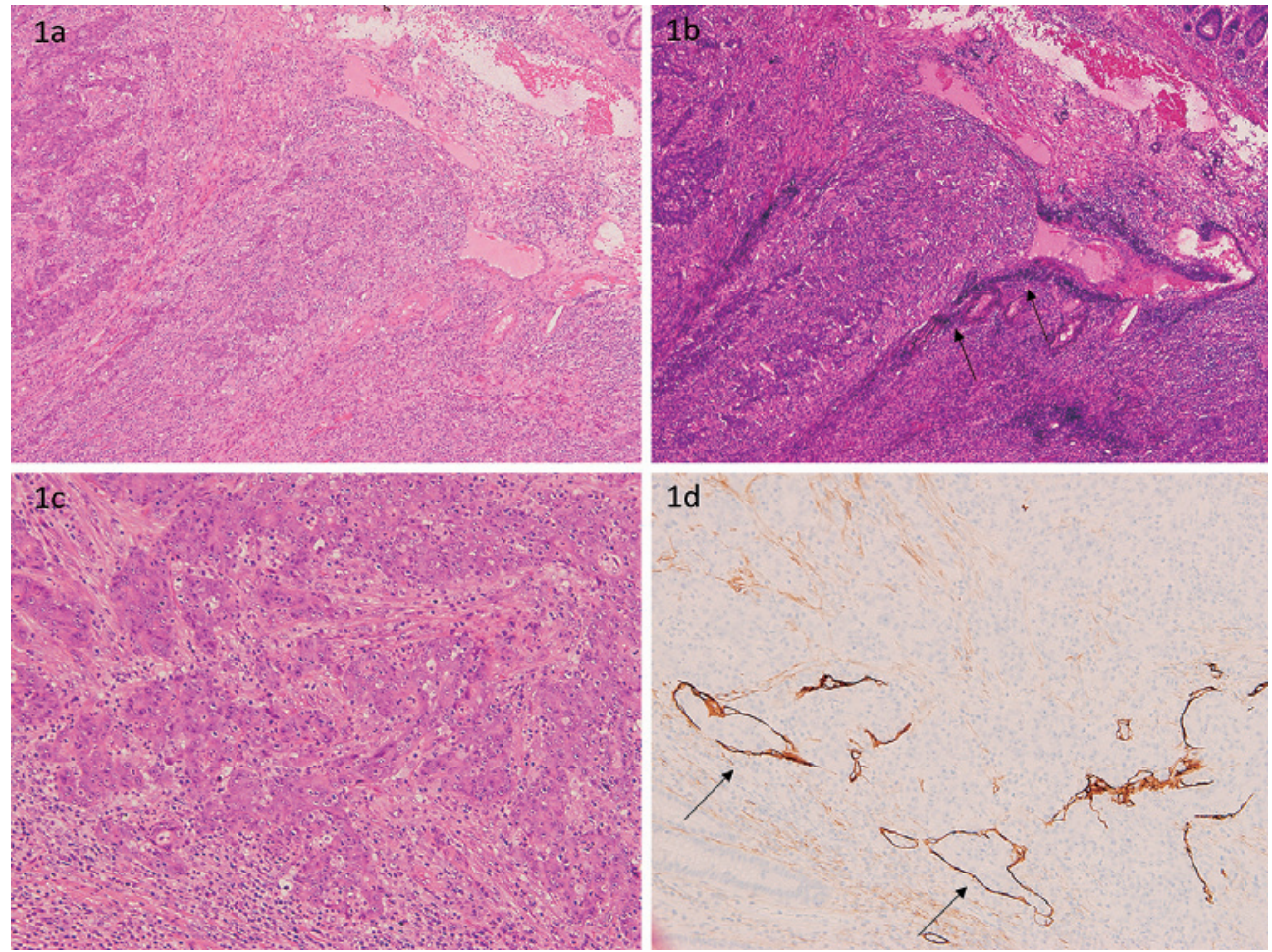

Figure 1. Immunohistochemical examination of lymphatic and venous invasion.

1a. Hematoxylin and eosin (HE) staining did not show venous invasion clearly in a T1 cancer case. 1b. Venous invasion was detected clearly with E-HE. 1c. HE staining did not show lymphatic invasion clearly in a T1 cancer case. 1d. Lymphatic invasion was detected clearly with D2-40 in the T1 cancer case.

and nuclei atypia, regardless of invasion to the lamina propria in Japan, whereas it was dysplastic cells invading the lamina propria in Korea. Regarding the definition of T1 cancer, depth of invasion was provided in Japan as the level of cancer cell infiltration or lymphatic and venous invasion, whereas in Korea it was provided as the level of cancer cell infiltration regardless of the depth of lymphatic and venous invasion. Histological types of tumors were classified as follows: well; moderately; or poorly-differentiated adenocarcinoma. Poorly-differentiated adenocarcinoma also included mucinous adenocarcinoma and signet ring cell carcinoma in this study. Lymphatic and venous invasion was examined using hematoxylin and eosin (HE) staining. Immunohistochemical examinations such as E-HE (elastic HE stain) for venous invasion and D2-40 for lymphatic invasion were performed in Japan (Figure 1). On the other hand, immunohistochemical examination such as ERG (ETS [erythroblast transformation-specific]-related gene) was performed for lymphatic and venous invasion in Korea. This study was a part of the subgroup analysis of a study approved by the Institutional Review Board of Kyoto Prefectural University of Medicine (IRB approval No. ERB-C-838-2) and the Konkuk University (IRB approval No. KUH1010644). This study was registered with the clinical trial registration sites in Ja- pan (http://umin.co.jp) as UMIN000019935 and in Korea (ht tp://cris.nih.go.kr) as KCT0001490.

\section{Statistical Analysis}

Statistical analyses were performed using the MannWhitney- $U$ and the chi-squared tests (SPSS version 22.0 for windows, IBM Japan, Ltd., Tokyo, Japan). Continuous variables, such as patient age and tumor size, were analyzed using the Mann-Whitney- $U$ test. Multivariate logistic regression analyses of the right-sided colon in $\mathrm{T} 1$ cancer were performed for related factors with a $P$-value $=0.1$ in univariate analysis. A $P$-value $<0.05$ was considered statistically significant.

\section{Results}

The differences of clinical characteristics in 726 Japanese cases and 149 Korean cases of early CRC are shown in Table 1. Median age was significantly higher in Japan than it was in Korea $(67.8 \pm 10.6$ years vs. $62.2 \pm 10.1$ years, $P<$ $0.001)$. The right-sided colon incidence $(38.7 \%$ vs. $22.1 \%, P$ $<0.001)$ was higher in Japan than it was in Korea. The rate of Tis in Japan was higher than that in Korea $(59.8 \%$ vs. 
Table 1. Differences in Characteristics of Tis and T1 CRCs between Two Countries.

\begin{tabular}{|c|c|c|c|}
\hline & Japan & Korea & P-value \\
\hline Case number & 726 & 149 & \\
\hline Age, mean \pm SD & $67.8 \pm 10.6$ & $62.2 \pm 10.1$ & $<0.001$ \\
\hline The rate of the elderly and the non-elderly, $\%$ (n) & $66.4 / 33.6(482 / 244)$ & $43.0 / 57.1(64 / 85)$ & $<0.001$ \\
\hline The rate of patients $\geq 75$ y.o., $\%$ (n) & $27.8(202)$ & $8.8(13)$ & $<0.001$ \\
\hline Sex, Male/Female, \% (n) & $62.4 / 37.6(453 / 273)$ & $64.4 / 35.6(96 / 53)$ & 0.710 \\
\hline Tumor size $(\mathrm{mm})$, mean $\pm \mathrm{SD}$ & $24.6 \pm 16.9$ & $21.9 \pm 13.8$ & 0.066 \\
\hline Tumor location, right-sided/left-sided/rectum, \% (n) & $38.7 / 31.3 / 30.0(280 / 226 / 217)$ & $22.2 / 45.0 / 32.9(33 / 67 / 49)$ & $<0.001$ \\
\hline Antithrombotics, \% (n) & $12.8(38 / 296)$ & $6.6(5 / 76)$ & 0.160 \\
\hline Initial therapeutic method, Surgery/Endoscopy, \% (n) & 23.3/76.7 (169/557) & $21.5 / 78.5(32 / 117)$ & 0.670 \\
\hline Histological type, well+mod/por, \% (n) & $99.2 / 0.7 / 0.1(720 / 5 / 1)$ & $96.6 / 2.7 / 0.7(144 / 4 / 1)$ & 0.050 \\
\hline Invasion depth, Tis/T1, \% (n) & $59.8 / 40.2(434 / 292)$ & $51.0 / 49.0(76 / 73)$ & 0.047 \\
\hline \multicolumn{4}{|l|}{ Complications, \% (n) } \\
\hline Overall & $5.4(39)$ & $8.7(13)$ & 0.114 \\
\hline Surgery & $12.4(21)$ & $6.3(2)$ & 0.314 \\
\hline Endoscopy & $3.2(18)$ & $9.4(11)$ & 0.002 \\
\hline
\end{tabular}

y.o.: years old, right-sided: cecum to transverse colon, left-sided: descending colon to sigmoid colon, well: well differentiated adenocarcinoma, mod: moderately differentiated adenocarcinoma, por: poorly-differentiated adenocarcinoma

Table 2. Characteristic Differences of 510 Tis CRCs between Two Countries.

\begin{tabular}{lccc}
\hline & Japan & Korea & P-value \\
\hline Case number & 434 & 76 & $61.8 \pm 9.3$ \\
Age, mean \pm SD & $68.8 \pm 10.2$ & $14.5 / 85.5(11 / 65)$ & 0.001 \\
Initial therapeutic method, Surgery/Endoscopy, \% (n) & $5.8 / 94.2(25 / 409)$ & $21.5 \pm 14.9$ & 0.013 \\
Tumor size (mm), mean \pm SD & $26.1 \pm 18.3$ & $26.3 / 40.8 / 32.9(20 / 31 / 25)$ & 0.026 \\
Tumor location, right-sided/left-sided/rectum, \% (n) & $41.8 / 29.3 / 28.9(181 / 127 / 125)$ & $97.4 / 2.6(74 / 2)$ & 0.059 \\
Histological type, well+mod/por, \% (n) & $99.8 / 0.2(433 / 1)$ & 0 & n.c. \\
Venous invasion, \% (n) & 0 & 0 & n.c. \\
Lymphatic invasion, \% (n) & 0 & $0(0 / 11)$ & n.c. \\
Lymph node metastasis, \% (n) & $0(0 / 25)$ & & 0 \\
\hline
\end{tabular}

right-sided: cecum to transverse colon, left-sided: descending colon to sigmoid colon, well: well differentiated adenocarcinoma, mod: moderately differentiated adenocarcinoma, por: poorly-differentiated adenocarcinoma, n.c.: not calculated

$51.0 \%, P=0.047)$, according to the difference of Tis definition. Regarding complications, the rate of complication due to endoscopy in Japan was lower than it was in Korea $(3.2 \%$ vs. $9.4 \%, P=0.002)$.

Differences in cancer characteristics of Tis for the 510 patients from Japan and Korea are shown in Table 2. The mean age was higher in Japan than it was in Korea (68.8 \pm 10.2 years vs. $61.8 \pm 9.3$ years, $P<0.001)$. The rate of endoscopic resection as an initial therapeutic method was also significantly higher in Japan than it was in Korea (94.2\% vs. $85.5 \%, P=0.013)$. The rate of right-sided colon lesions was higher in Japan than it was in Korea $(41.8 \%$ vs. $26.3 \%, P=$ 0.026).

Differences in T1 cancer characteristics for the 365 patients from the two countries are shown in Table 3. The mean age was higher in Japan than it was in Korea (66.4 \pm 11.0 years vs. $62.6 \pm 11.0$ years, $P=0.007)$. However, the rate of endoscopic resection as an initial therapeutic method was significantly lower in Japan than it was in Korea (50.7\% vs. $71.2 \%, P=0.002$ ). There was no significant difference regarding the rate of additional surgery after endoscopic resections between Japan and Korea. The rates of right-sided colon lesions $(34.1 \%$ vs. $17.8 \%, P=0.011)$ were higher in Japan than they were in Korea. With respect to histopathological evaluation, both venous invasion $(18.6 \%$ vs. $1.4 \%, P<0.001)$ and lymphatic invasion $(25.3 \%$ vs. $13.7 \%, P=0.042$ ) were higher in Japan than Korea (Figure 2). However, there was no significant difference between Japan and Korea in the rate of lymph node metastasis in surgical cases $(11.8 \%$ vs. $10.0 \%, P=0.999)$.

The difference between Japan and Korea of locations of tumors for T1 cancer in the elderly and the non-elderly are shown in Figure 3. In Japan, there was a significant difference in rate of right-sided lesions between the non-elderly 
Table 3. Characteristic Differences of 365 T1 CRCs in Two Countries.

\begin{tabular}{|c|c|c|c|}
\hline & Japan & Korea & P-value \\
\hline Case number & 292 & 73 & \\
\hline Age, mean $\pm \mathrm{SD}$ & $66.4 \pm 11.0$ & $62.6 \pm 11.0$ & 0.007 \\
\hline Initial therapeutic method Surgery/Endoscopy, \% (n) & $49.3 / 50.7(144 / 148)$ & $28.8 / 71.2(21 / 52)$ & 0.002 \\
\hline The rate of additional surgery after endoscopic resection, $\%$ (n) & $11.5(17 / 148)$ & $17.3(9 / 52)$ & 0.338 \\
\hline Tumor size $(\mathrm{mm})$, mean $\pm \mathrm{SD}$ & $22.1 \pm 14.1$ & $22.3 \pm 12.6$ & 0.890 \\
\hline Tumor location, right-sided/left-sided/rectum, \% (n) & $34.1 / 34.1 / 31.7(99 / 99 / 92)$ & $17.8 / 49.3 / 32.9(13 / 36 / 24)$ & 0.011 \\
\hline Histological type, well+mod/por, \% (n) & 98.3/1.4/0.3 (287/4/1) & 95.9/2.7/1.4 (70/2/1) & 0.212 \\
\hline Venous invasion, \% (n) & $18.6(52)$ & $1.4(1)$ & $<0.001$ \\
\hline Lymphatic invasion, \% (n) & $25.3(71)$ & $13.7(10)$ & 0.042 \\
\hline Lymph node metastasis, \% (n) & $11.8(19 / 161)$ & $10.0(3 / 30)$ & 0.999 \\
\hline
\end{tabular}

right-sided: cecum to transverse colon, left-sided: descending colon to sigmoid colon, well: well differentiated adenocarcinoma, mod: moderately differentiated adenocarcinoma, por: poorly-differentiated adenocarcinoma
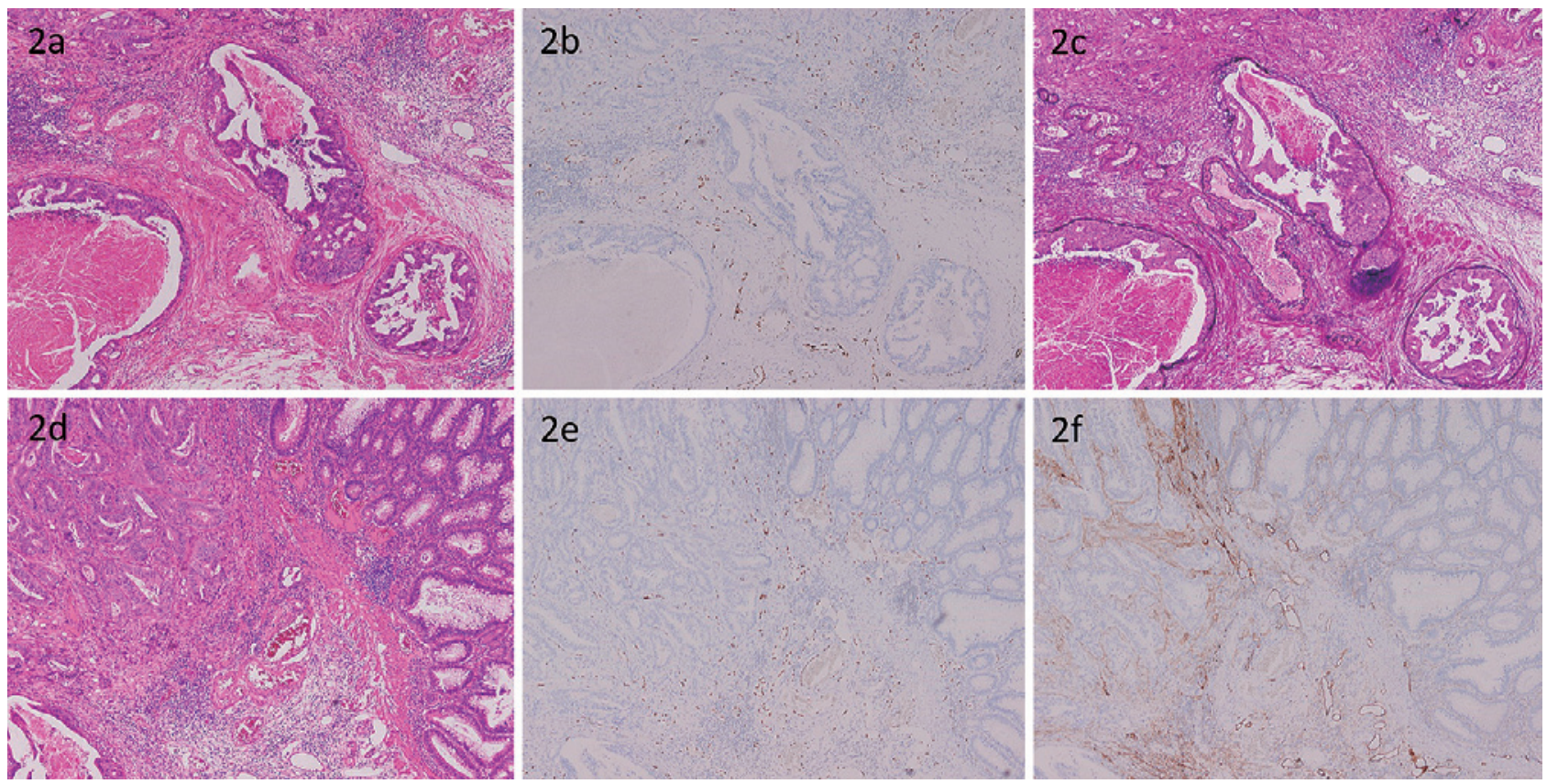

Figure 2. A case presentation of T1 cancer using immunohistochemical examination of EHE, D2-40, and ERG.

2a. Hematoxylin and eosin (HE) staining showed venous invasion. 2b. Venous invasion was detected clearly with ERG. It looked like accumulation of dots. 2c. E-HE staining showed venous invasion clearly. 2d. HE staining did not show lymphatic duct clearly. 2e. The existence of lymphatic duct was suspected with ERG; it looked like accumulation of dots. 2f. D2-40 staining clearly showed multiple lymphatic ducts without invasion. Lymphatic duct looked like a continuous circular line with D2-40.

and the elderly (26.0\% vs. $40.5 \%, P=0.010)$. However, there was no significant difference in this rate in Korea (10.8\% vs. $25.0 \%, P=0.201$ ).

Multivariate analysis showed that the factors related with the location of right-sided colon among $365 \mathrm{~T} 1 \mathrm{CRCs}$ were Japan (OR: 2.222, 95\%CI: 1.131-4.366, $P=0.021$ ) and older age (OR: $1.028,95 \% \mathrm{CI}: 1.005-1.052, P=0.015)$ (Table 4).

\section{Discussion}

In the current study, we analyzed Tis and T1 CRC characteristics in both Japan and Korea, especially focusing on histopathological managements such as definition of Tis and lymphatic and venous invasion. With respect to $\mathrm{T} 1$ cancer, the mean age, the right-sided colon incidence, and the rate of lymphatic and venous invasion were higher in Japan than they were in Korea.

Generally, previous studies regarding CRC have reported an increased proportion in Western countries in right-sided 


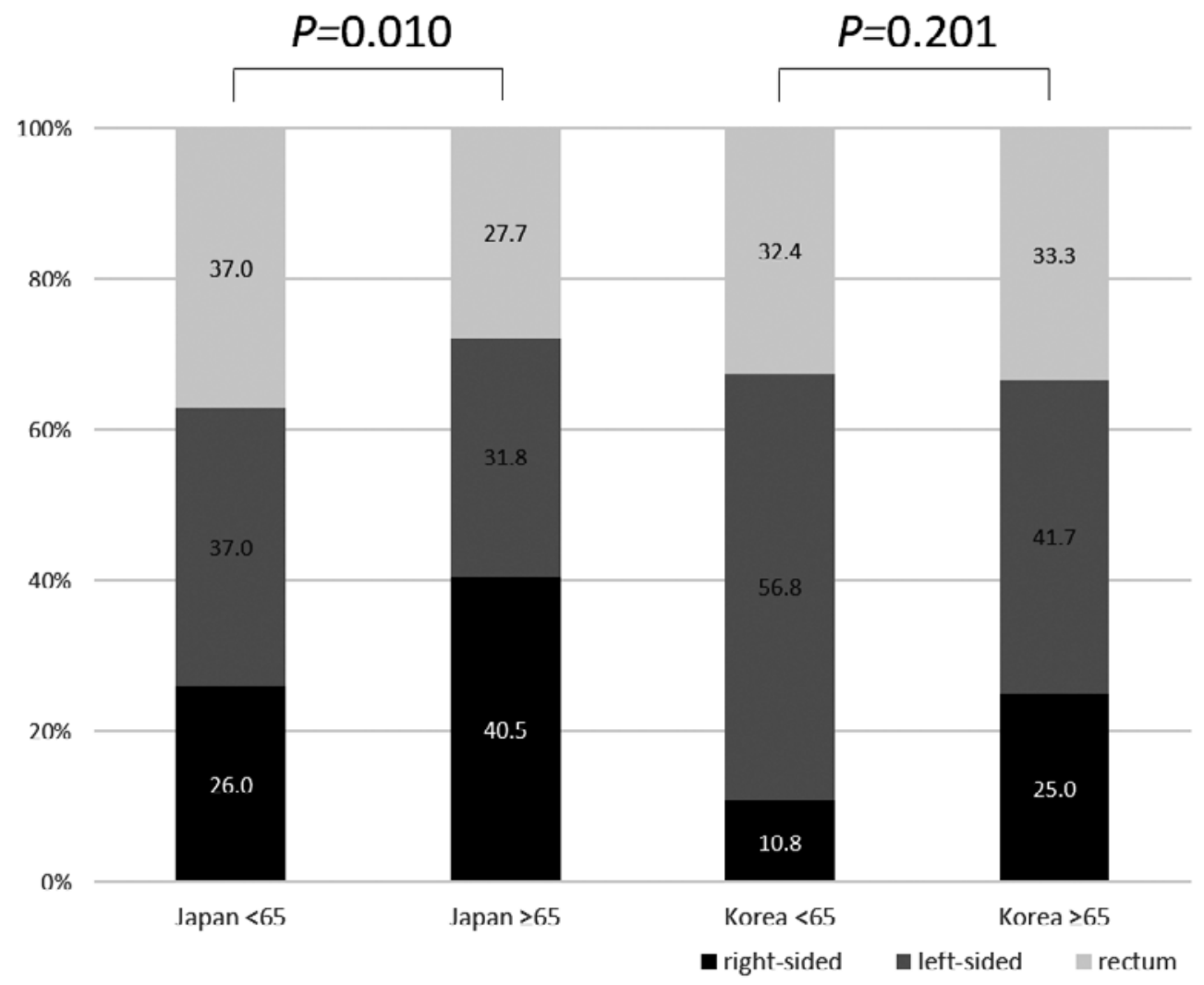

Figure 3. Analysis of tumor location of T1 CRCs between Japan and Korea for the elderly and the non-elderly.

CRC with increasing age ${ }^{19-23}$. Cooper et al. found in their study that the proportions of right-sided CRC were $36 \%$, $40 \%, 43 \%, 46 \%$, and $49 \%$, respectively, in patients $65-69$, $70-74,75-79,80-84$, and $\geq 85$ years old ${ }^{21}$. In Japan, the study by Okamoto et al. analyzed $196 \mathrm{CRCs}$, and reported that $15 \%, 21 \%, 32 \%, 42 \%$, and $57 \%$ of patients were $<50$, $50-59,60-69,70-79$, and $\geq 80$ years old ${ }^{24}$. In Korea, rightsided colon incidence of CRC also increased with aging ${ }^{25)}$. Most data in these studies were obtained from surgical operations in a single country. In our study, we showed that right-sided colon lesion proportions of $\mathrm{T} 1$ cancers were higher in Japan than they were in Korea. Additionally, a significant increase of right-sided colon lesion proportions between the non-elderly and the elderly was observed in Japan. However, this tendency was not observed in Korea. The different relationships between tumor location and aging for each country might be reasons for this difference. One of these possible reasons is that Japan has a larger elderly population than Korea; these Japanese data possibly enable us to predict future changes in Korea, although there are differences in histopathological evaluation, as described below. The other possible reason is that the differences of food habit, lifestyle and race might cause this increase in rightsided CRC in the elderly. However, there is a lack of Korean cases and this study was performed in only two institu- tions. Thus, further analyses should be performed for this.

Right-sided colon tumor has a relationship with a kind of cancer pathway. Two pathways have been suggested for sporadic CRC carcinogenesis: chromosomal instability (CIN); and microsatellite instability (MSI) ${ }^{26)}$. CIN is predominantly associated with the adenoma to carcinoma sequence. On the other hand, MSI is associated with the serrated neoplastic pathway $^{27}$. CIN is more frequent in cancers originating from the left-sided colon and rectum than in those originating from the right-sided colon ${ }^{26}$. By contrast, most sporadic MSI high CRCs tend to occur in the right-sided colon in the elderly $^{28}$. Thus, the increase in right-sided early CRC in the elderly may be due to genetic changes in the population in Japan. On the other hand, those changes may not be detected in Korean elderly populations.

As shown by the higher prevalence of Tis cancer in Japan $(59.8 \%)$ as opposed to in Korea $(51.0 \%)$ in our study findings, the small number of Korean cases might be due to the lower prevalence of Tis cancer based on the different histopathological definitions of Tis between the countries. In Korea, most of the endoscopically resected colorectal neoplasms are finally diagnosed as tubular adenoma with either high-grade or low-grade dysplasia instead of Tis cancer, according to the WHO criteria. Thus, "enlarged nuclei and prominent nucleoli regardless of invasion" is diagnosed as 
Table 4. Multivariate Analysis of Related Factors for Right-sided Colon of 365 T1 CRCs.

\begin{tabular}{|c|c|c|c|c|c|c|c|c|c|c|c|c|}
\hline \multirow[b]{4}{*}{ Case number } & & & & & \multicolumn{8}{|c|}{ Logistic regression analysis (right-sided colon $=1$, Other $=0$ ) } \\
\hline & \multicolumn{4}{|c|}{ Location } & \multicolumn{4}{|c|}{ Univariate } & \multicolumn{4}{|c|}{ Multivariate } \\
\hline & \multicolumn{2}{|c|}{ right-sided colon } & & \multirow[t]{2}{*}{ Other } & \multirow[t]{2}{*}{ OR } & \multicolumn{2}{|c|}{$95 \% \mathrm{CI}$} & \multirow[t]{2}{*}{ P-value } & \multirow[t]{2}{*}{ OR } & \multicolumn{2}{|c|}{$95 \% \mathrm{CI}$} & \multirow[t]{2}{*}{ P-value } \\
\hline & 112 & & 253 & & & & & & & & & \\
\hline \multicolumn{13}{|l|}{ Country } \\
\hline Japan & 99, & $88.39 \%$ & 193, & $76.28 \%$ & 2.369 & 1.240 & 4.524 & 0.009 & 2.222 & 1.131, & 4.366 & 0.021 \\
\hline Korea & 13 , & $11.61 \%$ & 60 & $23.72 \%$ & ref. & & & & & & & \\
\hline \multicolumn{13}{|l|}{ Sex } \\
\hline Male & 68 & $60.71 \%$ & 158 & $62.45 \%$ & ref. & & & & & & & \\
\hline Female & 44 & $39.29 \%$ & 95 & $37.55 \%$ & 1.076 & 0.682 , & 1.699 & 0.753 & - & & & \\
\hline \multicolumn{13}{|l|}{ Age } \\
\hline mean $\pm \mathrm{SD}$ & \multicolumn{2}{|c|}{$68.3 \pm 9.6$} & \multicolumn{2}{|c|}{$64.5 \pm 11.4$} & 1.035 & 1.012, & 1.058 & 0.002 & 1.028 & 1.005, & 1.052 & 0.015 \\
\hline median [IQR] & 70.0 & {$[62.00,75.00]$} & 65.0 & {$[58.50,72.00]$} & & & & & & & & \\
\hline \multicolumn{13}{|l|}{ Tumor size } \\
\hline mean $\pm \mathrm{SD}$ & \multirow{2}{*}{\multicolumn{2}{|c|}{$\begin{array}{l}22.9 \pm 13.4 \\
20.0[12.00,30.00]\end{array}$}} & \multirow{2}{*}{\multicolumn{2}{|c|}{$\begin{array}{l}21.7 \pm 13.9 \\
20.0[12.00,26.50]\end{array}$}} & 1.006 & 0.990 & 1.023 & 0.465 & - & & & \\
\hline median [IQR] & & & & & & & & & & & & \\
\hline \multicolumn{13}{|l|}{ Histological type } \\
\hline Well & 108 & $96.43 \%$ & 249 & $98.42 \%$ & ref. & & & & ref. & & & \\
\hline Por & 4 & $3.57 \%$ & 2 & $0.79 \%$ & 4.611 & 0.832 & 25.554 & 0.080 & 6.081 & 0.829 & 44.621 & 0.076 \\
\hline Others & 0 & $0.00 \%$ & 2 & $0.79 \%$ & n.c. & & & & n.c. & & & \\
\hline \multicolumn{13}{|c|}{ Lymph.node metastasis } \\
\hline 1 & 107 & $97.27 \%$ & 231 & $92.40 \%$ & ref. & & & & ref. & & & \\
\hline 2 & 3 & $2.73 \%$ & 18 & $7.20 \%$ & 0.360 & 0.104 & 1.248 & 0.107 & 0.253 & 0.060 & 1.068 & 0.061 \\
\hline 3 & 0 & $0.00 \%$ & 1 & $0.40 \%$ & n.c. & & & & n.c. & & & \\
\hline \multicolumn{13}{|c|}{ Lymphatic invasion } \\
\hline 0 & 188 & $76.73 \%$ & 85, & $77.98 \%$ & ref. & & & & & & & \\
\hline 1 & 44 & $17.96 \%$ & 17 & $15.60 \%$ & 0.855 & 0.462 & 1.581 & 0.617 & - & & & \\
\hline 2,3 & 13 & $5.31 \%$ & 7 & $6.42 \%$ & 1.191 & 0.459 & 3.091 & 0.720 & - & & & \\
\hline \multicolumn{13}{|l|}{ Venous invasion } \\
\hline 0 & 209 & $85.31 \%$ & 90 & $84.11 \%$ & ref. & & & & & & & \\
\hline 1 & 36 & $14.69 \%$ & 17 & $15.89 \%$ & 1.097 & 0.586 & 2.054 & 0.773 & - & & & \\
\hline
\end{tabular}

right-sided: cecum to transverse colon, IQR: interquartile range, well: well differentiated adenocarcinoma, por: poorly-differentiated adenocarcinoma, carcinoma, ref.: reference, n.c.: not calculated

Tis-staged CRC in Japan, but only as "adenoma with dysplasia" in Korea and the West, unless there is an invasion ${ }^{29)}$. Although there are WHO criteria for unifying histopathological evaluation in the world, each country has its own histopathological evaluation system. Yao et al. reported the international exchange like our study and the molecular analysis might be useful for the establishment of standardized diagnostic criteria of $\mathrm{CRC}^{29)}$. However, there is no reported detailed information about the difference between carcinoma in situ and intramucosal invasive cancer. In any future bilateral studies, the same histopathological evaluation should be performed.

There is one more histopathological finding about lymphatic and venous invasions. Previous reports revealed that histopathological evaluation of lymphatic invasion and venous invasion showed lower inter-observer variability ${ }^{30,31}$. Because recognizing veins and lymphatic channels using HE staining alone is difficult, immunohistochemical staining with the monoclonal D2-40 antibody reacts with the Olinked sialoglycoprotein (MW: $40 \mathrm{kDa}$ ) on the lymphatic endothelial surface and is used to distinguish lymphatic channels from small vessels ${ }^{32}$. Similarly, to identify venous walls, Elastica van Gieson (EVG), E-HE, or Victoria blue staining is used to stain and identify the venous wall elastic fibers, which stain dark violet using these techniques ${ }^{333}$. Suzuki et al. reported when evaluating T1 cancer histology that immunohistochemical staining techniques reduced interobserver variability ${ }^{34)}$. Additionally, EVG staining significantly increased the rate of positive $\mathrm{T} 1$ cancer venous invasion $(33.1 \%)$ as compared with HE staining (17.7\%). In our study, there was a significant difference in the rates of lymphatic and venous invasion between Japan and Korea (venous invasion: $18.6 \%$ vs. $1.4 \%$ and lymphatic invasion: $25.3 \%$ vs. $13.7 \%$ ). This might be due to the difference of types of immunohistochemical staining. Thus, E-HE and D2-40 were used in Japan. On the other hand, ERG was 
performed for all Korean cases. However, our study also showed that rates of lymph node metastasis were similar for both countries. It indicated that immunohistochemical staining might detect too many minor lymphatic and venous invasions in Japan. Generally, ERG stains the nucleus of endothelial cells and lymphatics and it looks like dots. The weak point of ERG is that it is considered not to be able to distinguish lymphatic and venous invasion from minute granulation tissue. On the other hand, D2-40 stains the cytoplasm of lymphatics and it looks like a continuous circular line. The weak point of D2-40 is to stain nervous tissue and desmoplastic reaction and increase false positive of lymphatic invasion. These differences with regard to histopathological evaluation in each country confuse international discussion about CRC in the world. A unified evaluation should be established in the future and further analysis should be performed to address this issue.

There were some limitations in this study. This was a retrospective study performed only in two institutions. Thus, it may include a bias and a standardized study examining our results should be performed in the future. Additionally, the Korean sample size was small due to the differences of histopathological evaluation, and there was the possibility of bias. Regarding histopathological evaluation, specimens in Korea and Japan were examined according to pathologic criteria in each country and by one or two pathologists in each country although all specimens should be pathologically examined according to the same pathologic criteria and by the same pathologist. It may influence the real difference in CRC between Japan and Korea.

In conclusion, our cross-national study showed several differences in Tis and T1 CRCs characteristics and histopathological evaluation in Korea and Japan. Additionally, researchers can know the difference of histopathological evaluation about intramucosal cancer and lymphatic and venous invasion in Japan and Korea, especially the difference between Japanese criteria and WHO criteria. We suggest close attention has to be paid to this kind of difference in a cross-national study. This kind of international study is thought to be useful to learn more about CRC features and decrease the incidence of colorectal cancer death.

\section{Acknowledgments}

We thank Dr. Takaaki Murakami, Dr. Ryohei Hirose, Dr. Kiyoshi Ogiso, Dr. Osamu Dohi, and all other members of the Department of Molecular Gastroenterology and Hepatology at the Kyoto Prefectural University of Medicine and the Department of Internal Medicine at Konkuk University School of Medicine for helping with this study.

\section{Conflicts of Interest}

There are no conflicts of interest.

\section{References}

1. Sano Y, Byeon JS, Li XB, et al. Colorectal cancer screening of the general population in East Asia. Dig Endosc. 2016 Feb; 28(3): 243-9.

2. World Cancer Research Fund International. Available from: http:// www.wcrf.org

3. Cancer Information Service by National Cancer Center. Cancer statics in Japan '16. Available from: https://ganjoho.jp/en/professio nal/statistics/bronchure/2016_en.html

4. Jung KW, Won YJ, Oh CM, et al. Cancer Statistics in Korea: Incidence, Mortality, Survival, and Prevalence in 2014. Cancer Research and Treatment. 2017 Mar; 49(2): 292-305.

5. Cabinet Office, Government of Japan. Available from: http://www 8.cao.go.jp/kourei/whitepaper/w-2015/html/gaiyou/s1_1.html.

6. Statistics Korea. Available from: http://kostat.go.kr/protal/eng/inde $\mathrm{X}$.action

7. Kim SY, Wie GA, Cho YA, et al. The role of red met and flavonoid consumption on cancer prevention: The Korean cancer screening examination cohort. Nutrients. 2017 Aug; 9(9): 938.

8. Wada K, Oba S, Tsuji M, et al. Meat consumption and colorectal cancer risk in Japan: The Takayma Study. Cancer Sci 2017 May; 108(5): 1065-70.

9. Cancer Facts and Figures 2013 in the Republic of Korea.

10. Song M, Garrett WS, Chan AT. Nutrients, foods, and colorectal cancer prevention. Gastroenterology. 2015 May; 148(6): 1244-60.

11. Yoshida N, Yagi N, Inada Y, et al. The ability of a novel blue laser imaging system for the diagnosis of colorectal polyps. Dig Endosc. 2014 May; 49(1): 250-8.

12. Sano Y, Ikematsu H, Fu KI, et al. Meshed capillary vessels by use of narrow-band imaging for differential diagnosis of small colorectal polyps. Gastrointest Endosc. 2009 Feb; 69(2): 278-83.

13. Kudo S, Kashida H, Tamura T, et al. Colonoscopic diagnosis and management of nonpolypoid early colorectal cancer. World J Surg. 2000 Sep; 24(9): 1081-90.

14. Tanaka S, Oka S, Kaneko I, et al. Endoscopic submucosal dissection for colorectal neoplasia: possibility of standardization. Gastrointest Endosc. 2007 Jul; 66(1): 100-7.

15. Saito Y, Uraoka T, Yamaguchi $Y$, et al. A prospective, multicenter study of 1111 colorectal endoscopic submucosal dissections (with video). Gastrointest Endosc. 2010 Oct; 72(6): 1217-25.

16. Yoshida N, Naito Y, Sakai K, et al. Outcome of endoscopic submucosal dissection for colorectal tumors in eldery people. Int $\mathrm{J}$ Colorectal Dis. 2010 Apr; 25(4): 455-61.

17. Japanese Society for Cancer of the Colon and Rectum, Japanese Classification of Colorectal Carcinoma, $2^{\text {nd }}$ edition. Tokyo, Japan: Kanehara \& Co., Ltd; 2009.

18. Hamilton SR, Aaltonen LA, editors. World Health Organization classification of tumors. Pathology and genetics of tumours of the digestive system. Lyon, France: IARC Press 2010: 104-9.

19. Slattery ML, Friedman GD, Poller JD, et al. A description of age, sex, and site distributions of colon carcinoma in three geographic areas. Cancer. 1996 Oct; 78(8): 1666-70.

20. Nelson RL, Dollear T, Freels $S$, et al. The relation of age, race, and gender to the subsite location of colorectal carcinoma. Cancer. 1997 Jul; 80(2): 193-7.

21. Cooper GS, Yuan Z, Landefeld CS, et al. A national populationbased study of incidence of colorectal cancer and age. Implications for screening in older Americans. Cancer. 1995 Feb; 75(3): 775-81. 
22. Stater G, Papatestas AE, Tartter PI, et al. Age distribution of rightand left-sided colorectal cancers. Am J Gastroenterol. 1982 Feb; 77(2): 63-6.

23. Fante R, Bebatti P, di Gregorio C, et al. Colorectal carcinoma in different age groups: a population-based investigation. Am J Gastroenterol. 1997 Sep; 92(9): 1505-9.

24. Okamoto M, Shiratori Y, Yamaji Y, et al. Relationship between age and site of colorectal cancer based on colonoscopy findings. Gastrointest Endosc. 2002 Apr; 55(4): 548-51.

25. Park HC, Shin A, Kim BW, et al. Data on the characteristics and the survival of Korean patients with colorectal cancer from the Korea central cancer registry. Ann Coloproctol. 2013 Aug; 29(4): 144-9.

26. Gervaz P, Bucher P, Morel P. Two colons-two cancers: paradigm shift and clinical implications. J Surg Oncol. 2004 Dec; 88(4): 261-6.

27. Leggett B, Whitehall V. Role of the serrated pathway in colorectal cancer pathogenesis. Gastroenterology. 2010 Jun; 138(6): 2088100 .

28. Kakar S, Burgart LJ, Thibodeau SN, et al. Frequency of loss of hMLH1 expression in colorectal carcinoma increases with advancing age. Cancer. 2003 Mar; 97(6): 1421-7.

29. Yao T, Shino S. Differences in the pathological diagnosis of colorectal neoplasia between the East and the West Present status and future perspectives from Japan. Dig Endosc. 2016 Apr; 28(3): 30611.

30. Schlemper RJ, Itabashi M, Kato Y, et al. Differences in the diagnostic criteria used by Japanese and Western pathologists to diagnose colorectal carcinoma. Cancer. 1998 Jan; 82(1): 60-9.

31. Schlemper RJ, Riddell RH, Kato Y, et al. The Vienna classification of gastrointestinal epithelial neoplasia. Gut. 2000 Aug; 47(2): 2515 .

32. Fogt F, Zimmerman RL, Ross HM, et al. Identification of lymphatic vessels in malignant, adenomatous and normal colonic mucosa using the novel immunostain D2-40. Oncol Rep. 2004 Jan; 11(1): 47-50.

33. Inoue $\mathrm{T}$, Mori M, Shimono $\mathrm{R}$, et al. Vascular invasion of colorectal carcinoma readily visible with certain stains. Dis Colon Rectum. 1992 Jan; 35(1): 34-9.

34. Suzuki A, Togashi K, Nokubi M, et al. Evaluation of venous invasion by Elastica van Gieson stain and tumor budding predicts local and distant metastases in patients with $\mathrm{T} 1$ stage colorectal cancer. Am J Surg Pathol. 2009 Nov; 33(11): 1601-7.

Journal of the Anus, Rectum and Colon is an Open Access journal distributed under the Creative Commons Attribution-NonCommercial-NoDerivatives 4.0 International License. To view the details of this license, please visit (https://creativ ecommons.org/licenses/by-nc-nd/4.0/). 